\title{
Numerical Solution of Time-Fractional Order Telegraph Equation by Bernstein Polynomials Operational Matrices
}

\author{
M. Asgari, ${ }^{1}$ R. Ezzati, ${ }^{1}$ and T. Allahviranloo ${ }^{2}$ \\ ${ }^{1}$ Department of Mathematics, Islamic Azad University, Karaj Branch, Karaj, Iran \\ ${ }^{2}$ Department of Mathematics, Islamic Azad University, Science and Research Branch, Tehran, Iran \\ Correspondence should be addressed to R. Ezzati; ezati@kiau.ac.ir
}

Received 27 May 2016; Revised 5 September 2016; Accepted 18 October 2016

Academic Editor: Zhen-Lai Han

Copyright (c) 2016 M. Asgari et al. This is an open access article distributed under the Creative Commons Attribution License, which permits unrestricted use, distribution, and reproduction in any medium, provided the original work is properly cited.

We present a new method to solve time-fractional order telegraph equation (TFOTE) by using Bernstein polynomials. By implementation of Bernstein polynomials operational matrices of fractional differential on TFOTE, we reduce the original problem to a linear system of algebraic equations. Also, we prove the convergence analysis. In order to show the efficiency of the proposed method, we present two numerical examples.

\section{Introduction}

Telegraph equations are hyperbolic partial differential equations that are applicable in modeling the reaction diffusion processes in various branches of engineering sciences and biological sciences. Those equations frequently arise in the study of wave propagation of electrical signal in a cable of transmission line and wave phenomena [1-4].

Many authors have used various numerical and analytical methods to solve the TFOTE. Chen and coworkers derived the analytical solution of the nonhomogeneous TFOTE by method of separation of variables [5]. Huang presented a new analytical solution for three basic problems of time-fractional telegraph equation. He solved Cauchy and signaling problems by Laplace and Fourier transforms and the boundary problem by spatial Sine transform [6]. Dehghan and Shokri developed a numerical method to solve the one-dimensional hyperbolic telegraph equation using the collocation points and approximated the solution by using thin plate spline radial basic functions [7]. Saadatmandi and Dehghan developed a numerical solution based on Chebyshev tau method [4]. Yousefi in [8] used Legendre multiwavelet Galerkin method for solving the hyperbolic telegraph equation. In [9], Das and Gupta used homotopy analysis method for solving fractional hyperbolic partial differential equation. In [10], Mollahasani et al. applied hybrid functions of Legendre polynomials and block pulse functions to obtain the solution of telegraph equation of fractional order.

In this paper, our study focuses on the time-fractional telegraph equation of order $2 \alpha$ :

$$
\begin{aligned}
& \frac{\partial^{2 \alpha} u(x, t)}{\partial t^{2 \alpha}}+a \frac{\partial^{\alpha} u(x, t)}{\partial t^{\alpha}}+b u(x, t) \\
& =c \frac{\partial^{2} u(x, t)}{\partial x^{2}}+f(x, t), \quad(x, t) \in[0,1] \times[0,1]
\end{aligned}
$$

with the initial and boundary conditions:

$$
\begin{gathered}
u(x, 0)=f_{1}(x), \\
u_{t}(x, 0)=f_{2}(x), \\
u(0, t)=g_{1}(t), \\
u(1, t)=g_{2}(t) .
\end{gathered}
$$

The right-hand-side function $f(x, t)$ is given, $1 / 2<\alpha \leq 1$, and also $a, b$, and $c$ are arbitrary positive constants. If $\alpha=1$, we have one-dimensional hyperbolic telegraph equation.

The rest of this paper is organized as follows: First, we present some preliminaries in fractional calculus. In Section 3, we briefly review some general concepts concerning Bernstein polynomials and the Bernstein polynomials 
operational matrix for fractional derivative. In Section 4, the method is applied to solve linear TFOTE. Section 5 exhibits an error estimation for the presented method. Section 6 illustrates two numerical examples to show the convergence and accuracy of the proposed method.

\section{Basic Definitions}

In this section, we present some basic definitions and properties of the fractional calculus which are going to be used in this paper.

Definition 1 (see [11]). The Riemann-Liouville fractional integral operator $I^{\alpha}$ of order $\alpha>0$ on the Lebesgue space $L^{1}[a, b]$ is given by

$$
\begin{aligned}
& \left(I_{0}^{\alpha} f\right)(t)=\left(I^{\alpha} f\right)(t)=\frac{1}{\Gamma(\alpha)} \int_{0}^{t}(t-\tau)^{\alpha-1} f(\tau) d \tau, \\
& \left(I^{0} f\right)(t)=f(t)
\end{aligned}
$$

where $\Gamma(\cdot)$ denotes Euler Gamma function.

Definition 2 (see [11]). The Caputo fractional derivative of order $\alpha$ is defined by

$$
\begin{array}{r}
D^{\alpha} f(t)=I^{n-\alpha} D^{n} f(t)=\frac{1}{\Gamma(n-\alpha)} \int_{0}^{t} \frac{f^{(n)}(\tau)}{(t-\tau)^{\alpha-n+1}} d \tau, \\
n-1<\alpha \leq n, n \in N,
\end{array}
$$

where $\alpha>0$ and $n$ is the smallest integer greater than $\alpha$. For the Caputo derivative, we have

$$
\begin{aligned}
D^{\alpha} x^{\beta} & = \begin{cases}\frac{\Gamma(\beta+1)}{\Gamma(\beta+1-\alpha)} x^{\beta-\alpha} & \beta \in N_{0}, \beta \geq\lceil\alpha\rceil, \\
0 & \beta \in N_{0}, \beta<\lceil\alpha\rceil,\end{cases} \\
D^{\alpha} c=0 \quad(c \in R) . &
\end{aligned}
$$

We use the ceiling function $\lceil\alpha\rceil$ to denote the smallest integer greater than or equal to $\alpha$ and $N_{0}=\{0,1,2, \ldots\}$. If $\alpha \in$ $N$, the Caputo differential operator coincides with the usual differential operator of an integer order.

For $n-1<\alpha \leq n, n \in N$, we have the following properties:

$$
\begin{aligned}
D^{\alpha} I^{\alpha} f(t) & =f(t), \\
I^{\alpha} D^{\alpha} f(t) & =f(t)-\sum_{k=0}^{n-1} f^{(k)}\left(0^{+}\right) \frac{t^{k}}{k !}, \\
t & >0 .
\end{aligned}
$$

\section{Bernstein Polynomials and Their Properties}

3.1. The Definition of Bernstein Polynomials Basis. The Bernstein polynomials (BPs) of degree $n$ on the interval $[0,1]$ are defined by

$$
B_{i, n}(x)=\left(\begin{array}{c}
n \\
i
\end{array}\right) x^{i}(1-x)^{n-i}, \quad 0 \leq i \leq n .
$$

These polynomials have the following properties on $[0,1]$ [12]:

(1) $B_{i, n}(x) \geq 0, i=0,1, \ldots, n, x \in[0,1]$,

(2) $\sum_{i=0}^{n} B_{i, n}(x)=1$,

(3) $B_{i, n}(x)=(1-x) B_{i, n-1}(x)+x B_{i-1, n-1}(x), i=0,1, \ldots, n$,

(4) $B_{i, n}(x)=\sum_{k=0}^{n-i}(-1)^{k}\left(\begin{array}{c}n \\ i\end{array}\right)\left(\begin{array}{c}n-i \\ k\end{array}\right) x^{i+k}, i=0,1, \ldots, n$.

Theorem 3 (see [13]). Suppose that $H=L^{2}[0,1]$ is a Hilbert space with the inner product and $Y=\operatorname{Span}\left\{B_{0, n}(x), B_{1, n}(x)\right.$, $\left.\ldots, B_{n, n}(x)\right\}$ is a finite dimensional and closed subspace; therefore, $Y$ is a complete subspace of $H$. So, if $f$ is an arbitrary element in $H$, it has a unique best approximation out of $Y$ such as $y_{0}$; that is,

$$
\left\|f-y_{0}\right\|_{2} \leq\|f-y\|_{2}, \quad \exists y_{0} \in Y \text { s.t. } \forall y \in Y,
$$

where $\|f\|_{2}=\sqrt{\langle f, f\rangle}$ and $\langle f, g\rangle=\int_{0}^{1} f(t) g(t) d t$. So, there exist unique coefficients $c_{0}, c_{1}, \ldots, c_{n}$ such that

$$
f(x) \simeq y_{0}=\sum_{i=0}^{n} c_{i} B_{i, n}(x)=c^{T} \varphi(x),
$$

where $c^{T}=\left[c_{0}, c_{1}, \ldots, c_{n}\right]$ and

$$
\varphi(x)=\left[B_{0, n}(x), B_{1, n}(x), \ldots, B_{n, n}(x)\right]^{T} .
$$

Lemma 4 (see [14]). If $\varphi(x)=\left[B_{0, n}(x), B_{1, n}(x), \ldots, B_{n, n}(x)\right]^{T}$ is a complete basis, then $\varphi(x)=A T_{n}(x)$, where $A$ is an $(n+$ $1) \times(n+1)$ upper triangular matrix with

$$
a_{i+1, j+1}= \begin{cases}(-1)^{j-i}\left(\begin{array}{l}
n \\
i
\end{array}\right)\left(\begin{array}{l}
n-i \\
j-i
\end{array}\right) & i \leqslant j, \\
0 & i>j,\end{cases}
$$

for $i, j=0,1, \ldots, n$ and $T_{n}(x)=\left[1, x, x^{2}, \ldots, x^{n}\right]^{T}$.

3.2. Function Approximation. A function $f(x, t) \in L^{2}([0,1] \times$ $[0,1])$ can be expressed in terms of the Bernstein polynomials basis as

$$
\begin{aligned}
f(x, t) & =\sum_{i=0}^{\infty} \sum_{j=0}^{\infty} f_{i j} B_{i, n}(x) B_{j, n}(t) \\
& \cong \sum_{i=0}^{n} \sum_{j=0}^{n} f_{i j} B_{i, n}(x) B_{j, n}(t)=\varphi^{T}(x) F \varphi(t),
\end{aligned}
$$

where

$$
F=\left[\begin{array}{cccc}
f_{00} & f_{01} & \cdots & f_{0 n} \\
f_{10} & f_{11} & \cdots & f_{1 n} \\
\vdots & \vdots & \ddots & \vdots \\
f_{n 0} & f_{n 1} & \cdots & f_{n n}
\end{array}\right]
$$


with

$$
f_{i j}=\frac{\left(\varphi_{i}(x),\left(f(x, t), \varphi_{j}(t)\right)\right)}{\left(\varphi_{i}(x), \varphi_{i}(x)\right)\left(\varphi_{j}(t), \varphi_{j}(t)\right)},
$$

$$
i, j=0,1, \ldots, n \text {. }
$$

\subsection{Operational Matrix for Fractional Derivative}

Theorem 5. Let $\varphi(x)$ be $n+1$ vector defined in (10); then,

$$
D^{\alpha} \varphi(x) \simeq A P E^{T} \varphi(x),
$$

where $A, P$, and $E$ are $(n+1) \times(n+1)$ matrices that $A$ is defined in (11); $P$ is a diagonal matrix with

$$
\begin{aligned}
& P_{i+1, i+1}=\frac{\Gamma(i+1)}{\Gamma(i+1-\alpha)}, \quad i=\lceil\alpha\rceil, \ldots, n, \\
& E=\left[E_{0}, E_{1}, \ldots, E_{n}\right], \\
& E_{i}=Q^{-1}\left[\bar{E}_{i 0}, \bar{E}_{i 1}, \ldots, \bar{E}_{i n}\right], \\
& \bar{E}_{i j}=\int_{0}^{1} x^{i-\alpha} B_{j n}(x) d x=\frac{n ! \Gamma(i+j-\alpha+1)}{j ! \Gamma(i+n-\alpha+2)}, \\
& i=\lceil\alpha\rceil, \ldots, n, \quad j=0,1, \ldots, n .
\end{aligned}
$$

$D^{\alpha}$ is called the Bernstein polynomials operational matrix of fractional derivative [14].

\section{Description of the Method}

Clearly, $u(x, t) \in L^{2}([0,1] \times[0,1])$ can be approximated by using Bernstein polynomials as

$$
u(x, t)=\varphi^{T}(x) U \varphi(t),
$$

where $\varphi(x)$ and $\varphi(t)$ are $(n+1)$ vectors defined in (10) and the unknown $U$ is $(n+1) \times(n+1)$ matrix that can be shown as

$$
U=\left[\begin{array}{cccc}
u_{00} & u_{01} & \cdots & u_{0 n} \\
u_{10} & u_{11} & \cdots & u_{1 n} \\
\vdots & \vdots & \ddots & \vdots \\
u_{n 0} & u_{n 1} & \cdots & u_{n n}
\end{array}\right] .
$$

Now, we can write

$$
\begin{aligned}
\frac{\partial^{2 \alpha} u(x, t)}{\partial t^{2 \alpha}} & =\frac{\partial^{2 \alpha}\left(\varphi^{T}(x) U \varphi(t)\right)}{\partial t^{2 \alpha}} \\
& =\varphi^{T}(x) U \frac{\partial^{2 \alpha}(\varphi(t))}{\partial t^{2 \alpha}} \\
& =\varphi^{T}(x) U D^{2 \alpha} \varphi(t), \\
u_{t}(x, t) & =\frac{\partial\left(\varphi^{T}(x) U \varphi(t)\right)}{\partial t}=\varphi^{T}(x) U D \varphi(t),
\end{aligned}
$$

$$
\begin{aligned}
\frac{\partial^{\beta} u(x, t)}{\partial x^{\beta}} & =\frac{\partial^{\beta}\left(\varphi^{T}(x) U \varphi(t)\right)}{\partial x^{\beta}} \\
& =\left[\frac{\partial^{\beta} \varphi(x)}{\partial x^{\beta}}\right]^{T} U \varphi(t) \\
& =\left[D^{\beta} \varphi(x)\right]^{T} U \varphi(t) \\
& =\varphi^{T}(x)\left(D^{\beta}\right)^{T} U \varphi(t) .
\end{aligned}
$$

Substituting (19) and (21) into (1), we have

$$
\begin{aligned}
\varphi^{T} & (x) U\left[D^{2 \alpha}+a D^{\alpha}+b I\right] \varphi(t) \\
& =c \varphi^{T}(x)\left(D^{2}\right)^{T} U \varphi(t)+f(x, t) .
\end{aligned}
$$

Now, we collocate $(22)$ in $(n-1) \times(n-1)$ Newton-Cotes nodes as

$$
\begin{aligned}
& x_{i}=\frac{2 i-1}{2(n+1)}, \\
& y_{j}=\frac{2 j-1}{2(n+1)},
\end{aligned}
$$

$$
i, j=2,3, \ldots, n \text {. }
$$

So, we have $(n-1)^{2}$ equations as

$$
\begin{aligned}
R\left(x_{i}, t_{j}\right)= & \varphi^{T}\left(x_{i}\right) U\left[D^{2 \alpha}+a D^{\alpha}+b I\right] \varphi\left(t_{j}\right) \\
& -c \varphi^{T}\left(x_{i}\right)\left(D^{2}\right)^{T} U \varphi\left(t_{j}\right)-f\left(x_{i}, t_{j}\right) \\
= & 0 .
\end{aligned}
$$

Applying (17) and (20) in the initial and boundary conditions (2), we get

$$
\begin{gathered}
u(x, 0)=\varphi^{T}(x) U \varphi(0)=f_{1}(x), \\
u_{t}(x, 0)=\varphi^{T}(x) U D \varphi(0)=f_{2}(x), \\
u(0, t)=\varphi^{T}(0) U \varphi(t)=g_{1}(t), \\
u(1, t)=\varphi^{T}(1) U \varphi(t)=g_{2}(t) .
\end{gathered}
$$

By collocating (25) in Newton-Cotes nodes $x_{i}$ and $y_{j}$ for $i=$ $1,2, \ldots, n+1$ and $j=1,2, \ldots, n-1$, we get $4 n$ equations. 
These equations together with (24) give $(n+1)^{2}$ equations, which can be solved for $u_{i j}, i, j=0,1, \ldots, n$. So, the unknown function $u(x, t)$ can be approximated.

\section{Convergence Analysis}

Theorem 6. Suppose that $f:[0,1] \times[0,1] \rightarrow R$ is a continuous function and all partial derivatives of $f(x, t)$ exist and are continuous. Let $Y=\operatorname{Span}\left\{B_{i n}(x) B_{j n}(t)\right\}, i, j=$ $0,1, \ldots, n$. If $c^{T} \varphi(x, t)=\sum_{i=0}^{n} \sum_{j=0}^{n} f_{i j} B_{i, n}(x) B_{j, n}(t) \in Y$ is the best approximation for $f$ out of $Y$ and also all $(n+1)$ th-order partial derivatives of $f$ are bounded in magnitude by $M$, then the error bound is presented as follows:

$$
\left\|f-c^{T} \varphi\right\|_{2} \leq \frac{2 M\left(K_{1}+K_{2}\right)^{n+2}}{(n+1) ! \sqrt{(2 n+3)(2 n+4)}},
$$

where $K_{1}=\max \left\{1-x_{0}, x_{0}\right\}$ and $K_{2}=\max \left\{1-t_{0}, t_{0}\right\}$.

Proof. By applying the Taylor expansion in two variables for $f(x, t)$, we have

$$
\begin{aligned}
& f(x, t)=f\left(x_{0}, t_{0}\right)+\left.\left(x-x_{0}\right) \frac{\partial f}{\partial x}\right|_{\left(x_{0}, t_{0}\right)} \\
& +\left.\left(t-t_{0}\right) \frac{\partial f}{\partial t}\right|_{\left(x_{0}, t_{0}\right)}+\frac{1}{2 !}\left[\left.\left(x-x_{0}\right)^{2} \frac{\partial^{2} f}{\partial x^{2}}\right|_{\left(x_{0}, t_{0}\right)}\right. \\
& \quad+\left.2\left(x-x_{0}\right)\left(t-t_{0}\right) \frac{\partial^{2} f}{\partial x t}\right|_{\left(x_{0}, t_{0}\right)}
\end{aligned}
$$

$$
\begin{aligned}
& \left.+\left.\left(t-t_{0}\right)^{2} \frac{\partial^{2} f}{\partial t^{2}}\right|_{\left(x_{0}, t_{0}\right)}\right]+\cdots+\frac{1}{n !}\left[\left(x-x_{0}\right) \frac{\partial}{\partial x}\right. \\
& \left.+\left(t-t_{0}\right) \frac{\partial}{\partial t}\right]^{n} f\left(x_{0}, t_{0}\right)+\frac{1}{(n+1) !}\left[\left(x-x_{0}\right) \frac{\partial}{\partial x}\right. \\
& \left.+\left(t-t_{0}\right) \frac{\partial}{\partial t}\right]^{n+1} f\left(x_{0}+\theta\left(x-x_{0}\right), t_{0}\right. \\
& \left.+\theta\left(t-t_{0}\right)\right)=f_{1}(x, y)+\frac{1}{(n+1) !}\left[\left(x-x_{0}\right) \frac{\partial}{\partial x}\right. \\
& \left.+\left(t-t_{0}\right) \frac{\partial}{\partial t}\right]^{n+1} f\left(x_{0}+\theta\left(x-x_{0}\right), t_{0}\right. \\
& \left.+\theta\left(t-t_{0}\right)\right), \quad 0 \leq \theta \leq 1 .
\end{aligned}
$$

Hence, from Taylor expansion, we have

$$
\begin{aligned}
& \left|f(x, t)-f_{1}(x, t)\right| \\
& =\frac{1}{(n+1) !}\left|\left[\left(x-x_{0}\right) \frac{\partial}{\partial x}+\left(t-t_{0}\right) \frac{\partial}{\partial t}\right]^{n+1} f(\eta, \xi)\right| .
\end{aligned}
$$

Since $c^{T} \varphi$ is the best approximation for $f$ out of $Y$, we conclude that

$$
\begin{aligned}
& \left\|f-c^{T} \varphi\right\|_{2}^{2} \leq\left\|f-f_{1}\right\|_{2}^{2}=\iint_{0}^{1}\left|f(x, t)-f_{1}(x, t)\right|^{2} d x d t=\iint_{0}^{1} \frac{1}{(n+1) !^{2}} \mid\left[\left(x-x_{0}\right) \frac{\partial}{\partial x}+\left(t-t_{0}\right) \frac{\partial}{\partial t}\right]^{n+1} \\
& \left.\cdot f(\eta, \xi)\right|^{2} d x d t=\iint_{0}^{1} \frac{1}{(n+1) !^{2}}\left|\sum_{r=0}^{n+1}\left(\begin{array}{c}
n+1 \\
r
\end{array}\right)\left(x-x_{0}\right)^{r}\left(t-t_{0}\right)^{n+1-r} \frac{\partial^{n+1}}{\partial x^{r} \partial t^{n+1-r}} f(\eta, \xi)\right|^{2} d x d t \\
& \quad \leq \frac{M^{2}}{(n+1) !^{2}} \iint_{0}^{1}\left|\sum_{r=0}^{n+1}\left(\begin{array}{c}
n+1 \\
r
\end{array}\right)\left(x-x_{0}\right)^{r}\left(t-t_{0}\right)^{n+1-r}\right|^{2} d x d t=\frac{M^{2}}{(n+1) !^{2}} \iint_{0}^{1}\left|\left[\left(x-x_{0}\right)+\left(t-t_{0}\right)\right]^{n+1}\right|^{2} d x d t \\
& \leq \frac{4 M^{2}\left(K_{1}+K_{2}\right)^{2 n+4}}{(n+1) !^{2}(2 n+3)(2 n+4)} .
\end{aligned}
$$

Clearly, we obtain the following result:

$$
\left\|f-c^{T} \varphi\right\|_{2} \leq \frac{2 M\left(K_{1}+K_{2}\right)^{n+2}}{(n+1) ! \sqrt{(2 n+3)(2 n+4)}} .
$$

Remark 7. Equation (26) shows that if $n \rightarrow \infty$, then $\| f-$ $c^{T} \varphi \|_{2} \rightarrow 0$.

\section{Numerical Examples}

To demonstrate the validity and applicability of the numerical scheme, we apply the present method for the following illustrative examples.

Example 1. Consider the time-fractional telegraph equation of order $2 \alpha$ :

$$
\frac{\partial^{2 \alpha} u(x, t)}{\partial t^{2 \alpha}}+20 \frac{\partial^{\alpha} u(x, t)}{\partial t^{\alpha}}+25 u(x, t)
$$


TABLE 1: The maximum absolute errors in Example 1.

\begin{tabular}{|c|c|c|c|c|}
\hline$x$ & $\frac{\alpha=1, n=24}{\text { ISF }}$ & $\alpha=1$ & $\alpha=.975$ & $\alpha$ \\
\hline 0.0 & $8.7 \times 10^{-3}$ & $8.673 \times 10^{-19}$ & $1.735 \times 10^{-18}$ & $8.674 \times 10^{-19}$ \\
\hline 0.1 & $2.9 \times 10^{-4}$ & $5.508 \times 10^{-5}$ & $5.550 \times 10^{-4}$ & $\times 10^{-3}$ \\
\hline 0.2 & $3.5 \times 10^{-4}$ & $5.172 \times 10^{-5}$ & $9.255 \times 10^{-4}$ & $\times 10^{-3}$ \\
\hline 0.3 & $3.1 \times 10^{-4}$ & & & \\
\hline 0.4 & $4.4 \times 10^{-4}$ & $1.688 \times 10^{-5}$ & $\times 10^{-3}$ & $4.7 \xi$ \\
\hline 0.5 & $3.4 \times 10^{-4}$ & $6.236 \times 10^{-6}$ & $1.921 \times 10^{-3}$ & $10^{-3}$ \\
\hline 0.6 & $2.5 \times 10^{-4}$ & $4.501 \times 1$ & $10^{-3}$ & 6.70 \\
\hline 0.7 & $2.3 \times 10^{-4}$ & $2.064 \times 10^{-5}$ & $10^{-3}$ & 7.08 \\
\hline 0.8 & $1.7 \times 10^{-4}$ & $3.993 \times 10^{-5}$ & $\times 10^{-3}$ & $10^{-3}$ \\
\hline 0.9 & $1.1 \times 10^{-4}$ & $4.562 \times 10^{-5}$ & $1.428 \times 10^{-3}$ & $4.382 \times 10^{-3}$ \\
\hline 1.0 & $4.0 \times 10^{-6}$ & $1.776 \times 10^{-15}$ & $2.109 \times 10^{-15}$ & $8.882 \times 10^{-16}$ \\
\hline
\end{tabular}

$$
\begin{aligned}
& \quad=\frac{\partial^{2} u(x, t)}{\partial x^{2}}+f(x, t), \\
& u(x, 0)=\sin (x), \\
& u_{t}(x, 0)=0, \\
& u(0, t)=0, \\
& u(1, t)=\cos (t) \sin (1), \\
& f(x, t)=-20 \sin (x) \sin (t)+25 \sin (x) \cos (t) .
\end{aligned}
$$

The exact solution of this example is $u(x, t)=\sin (x) \cos (t)$. In Table 1, we compare the obtained numerical results with the method of interpolating scaling functions operational matrix [15] (ISF), for $\alpha=1$. Also, in this table, we present the maximum absolute errors in $t=0.5$ for $n=4$ and various values of $\alpha$.

Example 2. Consider the time-fractional telegraph equation of order $2 \alpha$ :

$$
\begin{aligned}
& \frac{\partial^{2 \alpha} u(x, t)}{\partial t^{2 \alpha}}+40 \frac{\partial^{\alpha} u(x, t)}{\partial t^{\alpha}}+100 u(x, t) \\
& =\frac{\partial^{2} u(x, t)}{\partial x^{2}}+f(x, t), \\
& u(x, 0)=\sinh (x), \\
& u_{t}(x, 0)=-2 \sinh (x), \\
& u(0, t)=0, \\
& u(1, t)=e^{-2 t} \sinh (1), \\
& f(x, t)=23 e^{-2 t} \sinh (x) .
\end{aligned}
$$

Table 2 shows the absolute errors using the proposed method in the previous section for $\alpha=0.975$ and different values of $n$. The exact solution of this equation is $u(x, t)=$ $e^{-2 t} \sinh (x)$.
TABLE 2: The maximum absolute errors for $\alpha=0.975$.

\begin{tabular}{lccc}
\hline$x=t$ & $n=3$ & $n=4$ & $n=5$ \\
\hline 0.0 & $4.426 \times 10^{-4}$ & $8.630 \times 10^{-5}$ & $2.150 \times 10^{-6}$ \\
0.1 & $7.578 \times 10^{-5}$ & $6.734 \times 10^{-5}$ & $2.965 \times 10^{-5}$ \\
0.2 & $2.117 \times 10^{-5}$ & $1.172 \times 10^{-4}$ & $2.082 \times 10^{-4}$ \\
0.3 & $5.044 \times 10^{-4}$ & $1.851 \times 10^{-5}$ & $3.804 \times 10^{-4}$ \\
0.4 & $3.169 \times 10^{-4}$ & $3.180 \times 10^{-4}$ & $2.702 \times 10^{-4}$ \\
0.5 & $1.368 \times 10^{-3}$ & $1.011 \times 10^{-3}$ & $3.151 \times 10^{-4}$ \\
0.6 & $2.990 \times 10^{-3}$ & $1.997 \times 10^{-3}$ & $1.499 \times 10^{-3}$ \\
0.7 & $2.689 \times 10^{-3}$ & $3.270 \times 10^{-3}$ & $3.168 \times 10^{-3}$ \\
0.8 & $3.304 \times 10^{-2}$ & $6.917 \times 10^{-3}$ & $4.472 \times 10^{-3}$ \\
0.9 & $1.212 \times 10^{-1}$ & $2.302 \times 10^{-2}$ & $2.676 \times 10^{-3}$ \\
\hline
\end{tabular}

\section{Conclusion}

This paper proposed a numerical approach for solving TFOTE by using the operational matrices of Bernstein polynomials. The operational matrix of fractional derivative, $D^{\alpha}$, as well as collocation method was used to transform the TFOTE to a linear system of algebraic equations. The numerical results show that the proposed method in this paper can be a suitable method for solving these equations. To obtain the numerical results, we applied Mathematica 9 software.

\section{Competing Interests}

The authors declare that they have no competing interests.

\section{References}

[1] J. Banasiak and J. R. Mika, "Singularly perturbed telegraph equations with applications in the random walk theory," Journal of Applied Mathematics and Stochastic Analysis, vol. 11, no. 1, pp. 9-28, 1998.

[2] V. H. Weston and S. He, "Wave splitting of the telegraph equation in R3 and its application to inverse scattering," Inverse Problems, vol. 9, no. 6, pp. 789-812, 1993.

[3] P. M. Jordan and A. Puri, "Digital signal propagation in dispersive media," Journal of Applied Physics, vol. 85, no. 3, pp. $1273-1282,1999$.

[4] A. Saadatmandi and M. Dehghan, "Numerical solution of hyperbolic telegraph equation using the Chebyshev tau method," Numerical Methods for Partial Differential Equations, vol. 26, no. 1, pp. 239-252, 2010.

[5] J. Chen, F. Liu, and V. Anh, "Analytical solution for the timefractional telegraph equation by the method of separating variables," Journal of Mathematical Analysis and Applications, vol. 338, no. 2, pp. 1364-1377, 2008.

[6] F. Huang, "Analytical solution for the time-fractional telegraph equation," Journal of Applied Mathematics, vol. 2009, Article ID 890158, 9 pages, 2009.

[7] M. Dehghan and A. Shokri, "A numerical method for solving the hyperbolic telegraph equation," Numerical Methods for Partial Differential Equations, vol. 24, no. 4, pp. 1080-1093, 2008.

[8] S. A. Yousefi, "Legendre multiwavelet Galerkin method for solving the hyperbolic telegraph equation," Numerical Methods for Partial Differential Equations, vol. 26, no. 3, pp. 535-543, 2010. 
[9] S. Das and P. K. Gupta, "Homotopy analysis method for solving fractional hyperbolic partial differential equations," International Journal of Computer Mathematics, vol. 88, no. 3, pp. 578588, 2011.

[10] N. Mollahasani, M. Mohseni Moghadam, and K. Afrooz, "A new treatment based on hybrid functions to the solution of telegraph equations of fractional order," Applied Mathematical Modelling, vol. 40, no. 4, pp. 2804-2814, 2015.

[11] I. Podlubeny, Fractional Differential Equations, Academic Press, San Diego, Calif, USA, 1990.

[12] H. Jafari and H. Tajadodi, "Fractional order optimal control problems via the operational matrices of Bernstein polynomials," UPB Scientific Bulletin, Series A, vol. 76, no. 3, pp. 115-128, 2014.

[13] E. Kreyszig, Introductory Functional Analysis with Applications, John Wiley \& Sons, New York, NY, USA, 1978.

[14] M. Alipour, D. Rostamy, and D. Baleanu, "Solving multidimensional fractional optimal control problems with inequality constraint by Bernstein polynomials operational matrices," Journal of Vibration and Control, vol. 19, no. 16, pp. 2523-2540, 2012.

[15] M. Lakestani and B. N. Saray, "Numerical solution of telegraph equation using interpolating scaling functions," Computers \& Mathematics with Applications, vol. 60, no. 7, pp. 1964-1972, 2010. 


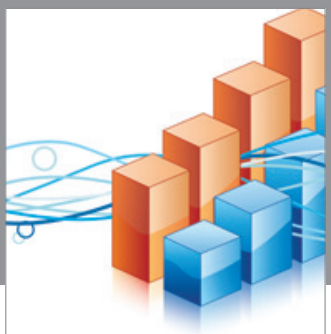

Advances in

Operations Research

vatem alat4

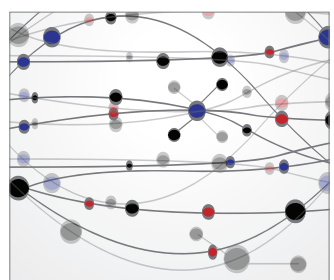

\section{The Scientific} World Journal
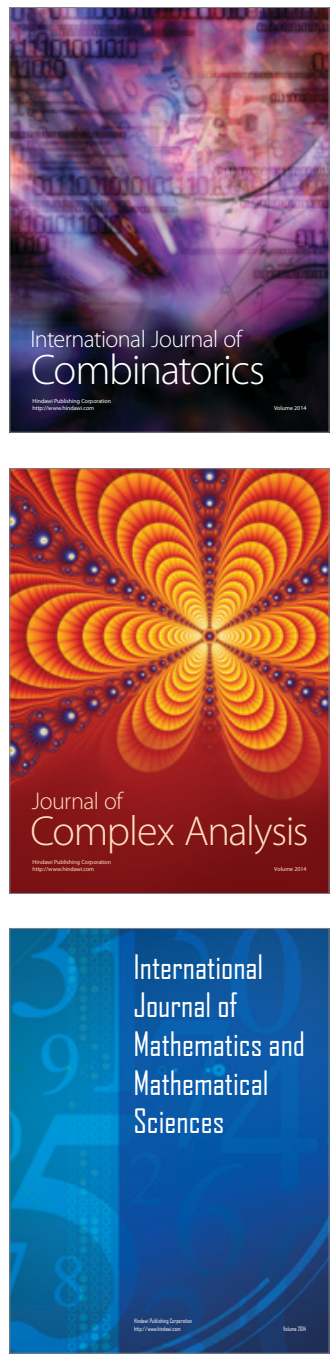
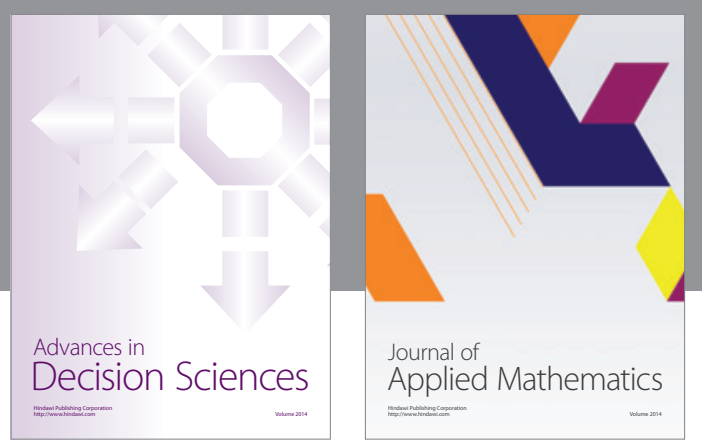

Algebra

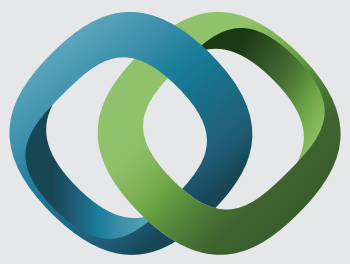

\section{Hindawi}

Submit your manuscripts at

http://www.hindawi.com
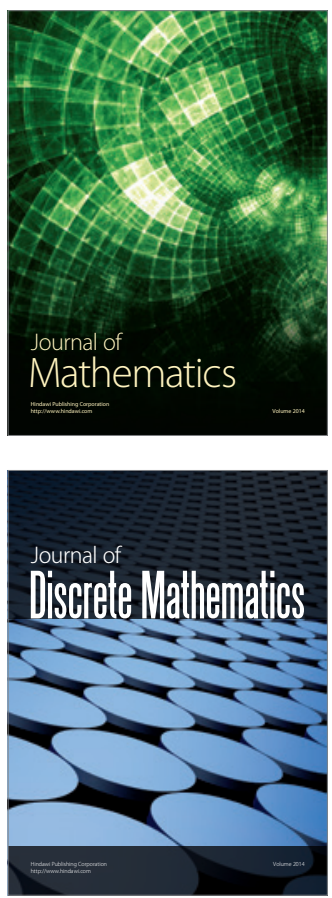



Mathematical Problems in Engineering


Journal of

Function Spaces

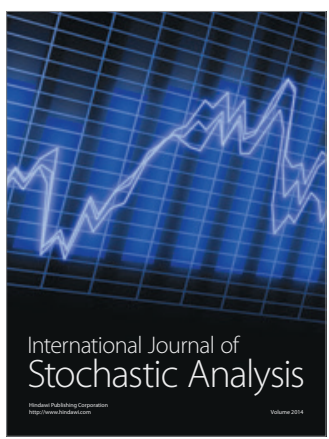

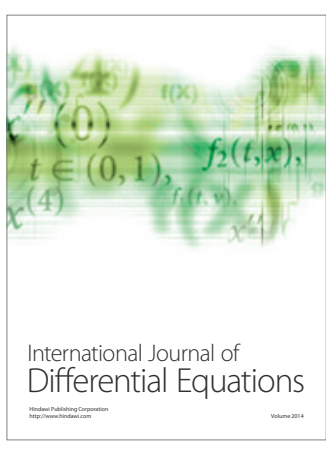
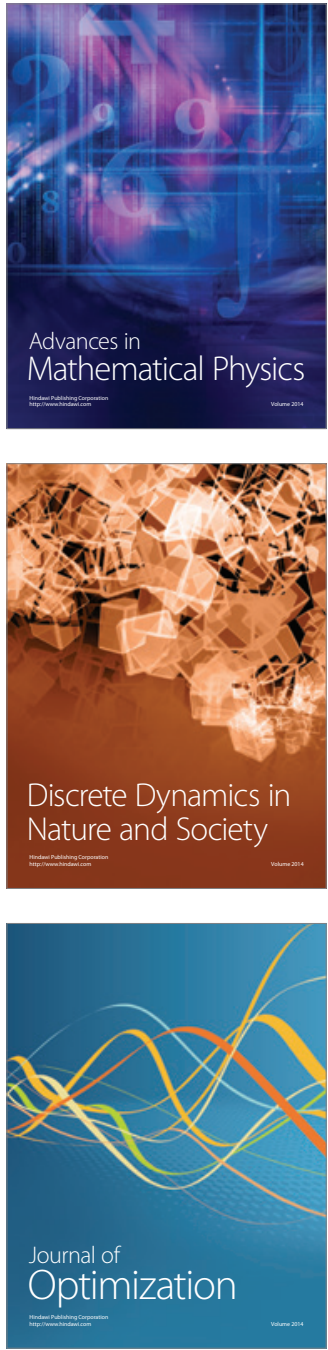